\title{
A PROGRAMMABLE CCD DRIVER CIRCUIT FOR MULTIPHASE CCD ORERATION
}

\author{
Audrey J. Ewin and Kenneth V. Reed \\ Code 724, NASA/Goddard Space Flight Center \\ Greenbelt, MD 20771
}

\section{Introduction}

A programmable $C C D$ driver circuit was designed to drive CCD's in multiphased modes. The purpose of the drive electronics was to operate developmental CCD imaging arrays for NASA's Moderate Resolution Imaging spectrometer - Tiltable (MODIS-T).

Five prototype arrays were designed with size and density specifications shown in Table 1. Valid's Graphics Editor (GED ${ }^{\mathrm{TM}}$ ) was used to design the driver. With this driver design, any of the five arrays can be readout. The CCD's are tested in the Detector Test Facility (DTF) - NASA/GSFC. Figure 1 shows the block diagram of the driver circuit with CCD and DTF interface signals. The START signal begins the driving operation at a known event in the frame readout sequence.

Five objectives for the driver were kept in mind during its design. The circuit:

1) Drives CCD electrode voltages between $0 \mathrm{~V}$ and $+30 \mathrm{~V}$ to produce reasonable potential wells.

2) Drives the CCDs in 4, 8, or 16 phase clocking modes.

3) The driving sequence is started with one input signal.

4) Allows programming of frame sequences required by arrays of any size.

5) Produces interfacing signals for the CCD and the DTF.

Designing the driver with $G D^{\mathrm{TM}}$ allowed functional simulation, timing verification, and certain packaging analyses to be done on the design before fabrication. Simulation of
Table 1: CCD Array Parameters

\begin{tabular}{|c|c|c|c|c|}
\hline$\overline{\overline{\text { Array }}}$ & Electrode & $\overline{\overline{\text { Pixel }}}$ & Clocking & $\overline{\text { Array }}$ \\
\hline \# & $\operatorname{Size}(\mu \mathrm{m})$ & ize $(u m)$ & Phases & Format \\
\hline 1 & 13 & 208 & 16 & $1 \times 16$ \\
\hline 2 & 13 & 208 & 16 & $4 \times 16$ \\
\hline 3 & 26 & 208 & 8 & $1 \times 16$ \\
\hline 4 & 52 & 208 & 4 & $1 \times 16$ \\
\hline 5 & 13 & 52 & 4 & $4 \times 32$ \\
\hline
\end{tabular}

the driver verified its function with the master clock running up to $10 \mathrm{MHz}$. This suggests a maximum rate of $400 \mathrm{Kpixels} / \mathrm{sec}$. Timing and packaging parameters were verified. The design uses 54 TTL component chips.

Two versions of hardware were fabricated: wirewrap and printed circuit board. Both were verified functionally with a logic analyzer. Their master clock's highest operating frequencies were found to be $1 \mathrm{MHz}$ and $10 \mathrm{MHz}$, respectively. Both versions of hardware have successfully driven the prototype detector arrays. Data obtained from the driven arrays is being used to produce specifications for a flight version MODIS-T CCD array.

\section{Design Method}

The MODIS-T CCD Imagers

From Table 1 it can be noted that four of the five MODIS-T CCDs have 208 square micron pixels. This unusually large pixel size forces the devices to have 4,8 , and 16

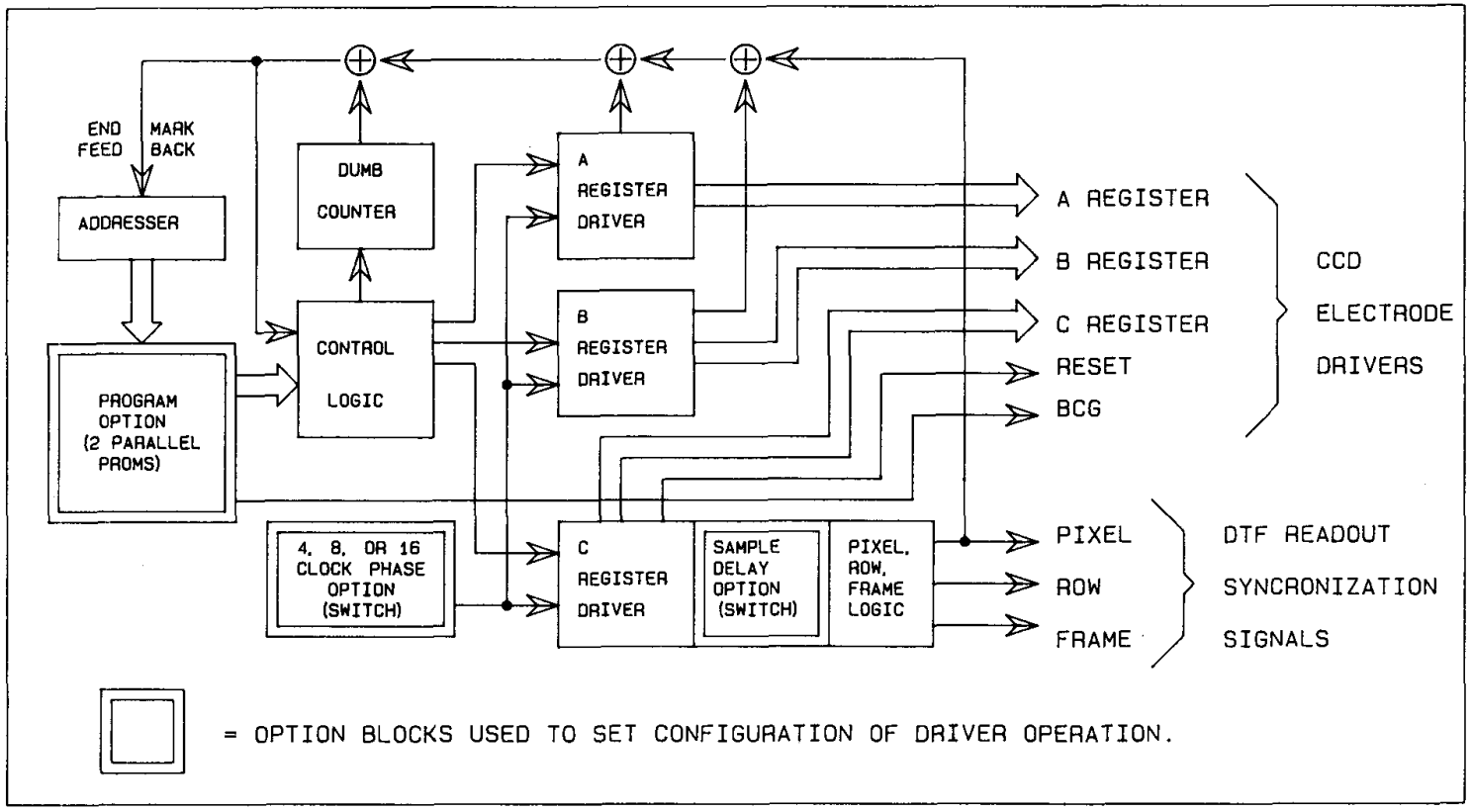

FIGURE 1: BLOCK DIAGRAM OF PROgRAMMABLE DRIVER FOR MULTIPHASE CCD OPERATION NOTE: CLK AND START SIGNALS ARE NOT SHOWN - THEY ARE INPUTS TO ENTIRE DRIVER CIRCUIT 
phases, respectively, with electrode widths of 52,26 , and 13 microns. The variations in phases and array format demands flexibility in the driver.

The MODIS-T devices are buried channel CCDs that require positive electrode voltages to create potential wells. Each pixel well is defined by applying $0 \mathrm{~V}$ on the two end electrodes with all electrodes in between at $+\mathrm{V}$ (usually between $+5 \mathrm{~V}$ and $+30 \mathrm{~V}$ ). This is true for all three phase modes.

The transfer of charge in a CCD occurs from clocking electrodes across the device. [1] A rotation is the clocking of electrodes required to transfer a charge packet from one element location to the next.

Inserting stretches of dead time between rotations makes following the CCD operation easier. Dead time is the event where all voltages are held steady for a predetermined span of time.

The arrays that were designed for MODIS-T are frame transfer devices [2]. Each array has three $C C D$ registers ( $A=$ image, $B=$ storage, and $C=o u t p u t$ ) and one output sensing node. The registers have to rotate independentiy from one another. The serial output register (C register) has one more pixel in its row, by the sensing node, than either the imaging or storage registers.

The entire sequence of events for one frame consists of alternating between rotation of at least one register and the insertion of dead time. The CCD layout requires four specific events for operation. They are: $A$ register transferring to $B$ register, B register transferring to $C$ register, $C$ register transferring to the output node, and dead time. By ordering the events in certain sequences, images created in any of the MODIS-T CCD's can be read from their output nodes.

\section{The CCD Driver}

The two logic blocks in the driver circuit that produce rotations and dead times, are the Register Driver and the Dumb Counter, respectively (Figure 1). These two blocks produce events on a one-time, on-command basis. Because of the three independently driven $C C D$ registers in each array, the driver actually has three identical Register Drivers. The commands that control the Register Drivers and Dumb Counter logic come from the Control Logic block. Also controlling Register Driver operation is the Clock Phase Option block.

The heart of the driver circuit is a frame sequence program in the Program option block. The program is a sequence of hexadecimal codes representing the exact order of events that will obtain the image data of one frame. The codes are broken down into control bits that are converted to commands within the control Logic block. In turn, decode logic within the Register Drivers and the Dumb Counter produce an END mark at the end of their respective event. This increments the Addresser to the next event in the sequence.

The driver operates in a continuous loop between issuing commands and producing events. The interface signals are by-products of the Program option block and the C Register Driver. At the end of the frame sequence program a designated bit is activated that starts the program over again.

\section{The Design}

This section describes the individual logic blocks that make up the driver design.

\section{Register Driver Logic and} Clock Phase Option

The Register Drivers perform the task of clocking and driving the appropriate voltages to the CCD register electrodes. There are three Register Driver blocks in the driver circuit; one for each $C C D$ register.

A Register Driver uses four 5194 shift registers to implement the 16 clocked signals. During initialization the correct 16 bit pattern for the desired number of phases is loaded into the S194s. The number of phases is set with two toggle switches in the Clock Phase option block. The bit patterns for 4 , 8 , and 16 phase operation are $(0110,0110,-$ $0110,0110\},\{0111,1110,0111,1110\}$, and $\{0111$,$1111,1111,11101$, respectively. Obviously, when using these signals, the 4,8 , and 16 phase configurations use 4, 8, and 16 lines of the possible 16 signal lines available.

After initialization, the loaded values are kept steady. The block is activated for rotation by sending the shift registers a command to start shifting. Near the end of rotation, two bits are used to create an END mark. This mark is sent back to the control Logic block to reset the rotate command for that Register Driver. The rotation stops until the next rotate command is received for that Register Driver.

Part of the command that the Register Driver receives is a decode control signal. It controls which Register Driver's END mark is allowed to advance the Addresser. This prevents conflicting END marks when two CCD registers rotate simultaneously.

All 16 bits for each $C C D$ register are driven with 5407 buffers. (ALS367 buffers were used for simulation.) The 5407 devices are open collector amplifiers with their outputs pulled up to $+\mathrm{V}$ through $1 \mathrm{k} \Omega$ resistors. The outputs reflect the $+V$ voltage for logic "1" while remaining $0 \mathrm{~V}$ for logic "0". The voltage $+\mathrm{V}$ can be set between $0 \mathrm{~V}$ and $+30 \mathrm{~V}$ giving flexibility in producing the optimum potential wells.

\section{Dumb Counter Logic}

The Dumb Counter is used to inject dead time between rotation events. It does so by counting up from zero to a predetermined value and then advances the Addresser. The block is comprised primarily of a single $\$ 169$ up/down counter.

During initialization and a rotation the Dumb Counter is not being used. During these times it will always be loading zeros into the S169. This keeps it from counting and makes it ready to produce a dead time when a command is received.

on command, the Dumb counter starts counting up until its output signal (gated by a decode control signal) produces an END mark. This reloads the counter with zeros and advances Addresser. The Dumb Counter is then ready to produce another dead time if asked to. The logic allows the driver to run a program calling for successive dead time periods.

The amount of dead time the driver 
produces is hard-wired. The period of the dead time is 16 clock cycles. Successive dead time commands keeps all output signals steady in time multiples of 16 clock cycles.

\section{Sample Delay Option and}

Pixel, Row, Frame Logic

A pixel is read out every time the $C$ register is rotated to the output sense node. The output voltage resulting from charge placed on the sense node represents the number of electrons in the charge packet. The DTF's $A / D$ converter must know when the output voltage is valid to take an accurate reading. The PIXEL signal serves this purpose. Every time the $C$ register rotates a PIXEL pulse follows.

A pulse on the PIXEL line that is sent to the DTF must occur after the rotating action of the $C$ register stops. Delaying the END mark from the $C$ Register Driver by two clock cycles through D flip-flops produces a pulse one clock cycle after the rotation stops.

The amount of settling time required for the entire charge packet to reach the sense node is largely determined by fabrication process of the device. To allow flexibility in finding the optimum settling time, the signal is delayed 6 more times. The Sample Delay option is a rotary switch that can be set to the terminal from the desired flip-flop output. The choices are a PIXEL pulse one, three, or seven clock cycles after rotation stops. Since this signal should occur before the next event begins, the chosen PIXEL signal, instead of the original END mark, is sent back to increment the Addresser.

The FRAME and ROW signals allow synchronization of the analog data with the DTF data acquisition program. Pixels at the beginning of a row have a Row pulse synchronized with the PIXEL pulse. Pixels that are at the beginning of a frame have synchronized FRAME, ROW, and PIXEL pulses. With these signals, imaging is accomplished. The FRAME and ROW signals are produced by gating the PIXEL signal for the appropriate pixel.

\section{Addresser Logic}

The Addresser keeps track of the current event in the frame sequence program. It is implemented with four 5169 counters that are cascaded to produce 16 address bits. The number of address bits provided determines the number of events available for a frame sequence program. If all 16 bits of the design were used then FFFF events can be programmed in the memory devices. This indicates the size of $C C D$ arrays the driver is capable of operating. This design version uses 12 of the 16 available address lines. During initialization and in the last event of the frame sequence program the counters are loaded with zeros. This allows a return to the first event in the program. The Addresser uses END marks to toggle the counters' clock inputs. The address is incremented by one at the end of every event.

\section{Program Option Logic}

The Program Option block contains the two memory devices used to hold the frame sequence program. The memory devices are configured in parallel to allow up to 16 bits of control signals. Beginning at address 0 , each location in the memory represents an event in the frame sequence. The output lines ( 8 program bits per device) are the signals used to control the rotations and dead times in the sequence. Table 2 lists the program bits and their purposes. Note the BCG signal comes right from program bit \#10. Like the other CCD signals it is driven with a 5407 buffer.

The program is carefully written to allow only one END mark to increment the Addresser during any event. This eliminates possible misfiring of the Addresser when two CCD registers rotate simultaneously.

The 27S43A PROMs were used for simulation of the driver. The hardware uses AM2864ADC EEPROMs to allow reprogramming of the frame sequence. Nonerasable PROMS are used to increase the frequency of operation.

\section{Control Logic}

The Control Logic block is used as an interface between the Program option block and the rest of the driver circuit. It modifies the program bits to produce proper control signals for the Register Drivers and the Dumb Counter.

Each Register Driver has its own control logic within the control Logic block. The Register Driver control logic is based on an S112 JK flip-flop that is configured as an SR flip-flop. This arrangement implements the time critical actions of starting (set) and stopping (reset) Register Driver shifting for a complete rotation. The flip-flop is set from a program bit changing from low to high. It is reset with the END mark of the

Table 2: Program Bits and Their Functions

\begin{tabular}{|c|c|c|c|}
\hline$\overline{\mathrm{PROM}}$ & Bit \# & Signal & Function \\
\hline \multirow{8}{*}{ A } & 1 & OUT $\langle 1\rangle$ & Gates A-RD's END mark to advance the Addresser \\
\hline & 2 & OUT $<2>$ & Commands $\mathrm{A}-\mathrm{RD}$ to rotate \\
\hline & 3 & OUT $<3>$ & Gates B-RD's END mark to advance the Addresser \\
\hline & 4 & OUT $\langle 4\rangle$ & Commands $\mathrm{B}-\mathrm{RD}$ to rotate \\
\hline & 5 & OUT $<5>$ & Gates C-RD's END mark to advance the Addresser \\
\hline & 6 & OUT $\langle 6\rangle$ & Commands $\mathrm{C}-\mathrm{RD}$ to rotate \\
\hline & 7 & OUT $<7>$ & Gates DC's END mark to advance the Addresser \\
\hline & 8 & OUT $<8>$ & Commands DC to count a span of dead time \\
\hline & 1 & OUT $<9>$ & Loads the Addresser with zeros \\
\hline & 2 & OUT $<10>B C G$ & the BCG CCD electrode \\
\hline B & 3 & $\mathrm{OUT}<11>\mathrm{R}$ & Gates the PIXEL signal to the ROW signal \\
\hline & $5-8$ & $\mathrm{OUT}<12>\mathrm{F}$ & $\begin{array}{l}\text { Gates the PIXEL signal to the FRAME signal } \\
\text { Unused }\end{array}$ \\
\hline
\end{tabular}

Note: $\mathrm{RD}=$ Register Driver, $\mathrm{DC}=$ Dumb Counter 
corresponding Register Driver, indicating the end of a rotation. This logic also produces the control signals to load the electrode bit pattern during initialization.

The output signal RESET is a CCD control signal that is conveniently tapped from the Control Logic block. It comes from the control logic for the $C$ Register Driver because it occurs before every $C$ register rotation. This signal is normally zero and pulses high a clock cycle before the $C$ Register Driver rotates. Of course, the RESET signal is also driven with a 5407 buffer. The signals to control the Dumb Counter only need to be latched through $D$ flip-flops to do their jobs.

\section{CLK and START Signals}

The CLK and START signals are special because they play a role in all major logic blocks of the driver. The CLK signal is the master clock for the driver. This signal determines the clocking speed of the phases and, ultimately, everything else. The oscillator output is divided down through two 74107A JK flip-flops to make a stable CLK signal. This gives a CLK with a frequency that is 4 times less than the oscillator's. The 107A allows up to 30 TTL loads to be driven.

The START signal is the only input signal to the driver. It is a single TTL line that goes high to initialize the driver and goes low to start the driving sequence. This signal needs only to be latched through a $107 \mathrm{~A}$ flip-flop to drive all the required loads. The clock input of this flip-flop is CLK.

\section{Modes of Operation}

There are three parameters of the driver that can be altered to tailor it to individual CCD needs. They are: number of phases, sample delay, and frame sequence program.

\section{Number of Phases}

The driver can operate in 4,8 , or 16 phase modes. The number of phases is set with two double-pole-double-throw switches. The switches control the specific bit pattern loaded into the Register Drivers' shift registers during initialization. The exact patterns are described in The Design section of this paper.

\section{Sample Delay}

The sample time is the time allowed after $C$ register rotation that a sample of the output is taken. The delay option allows adjustment of the amount of time after the $C$ register rotates that a pulse on the PIXEL line occurs. This option is set using a rotary switch with inputs from three different D flip-flop outputs. The flip-flops are in a line of eight through which the pixel pulse is passed. The delay times to choose from are a pixel pulse one, three, or seven clock cycles after $\mathrm{C}$ register rotation stops.

\section{Frame Sequence Program}

Perhaps the most versatile aspect of the CCD driver is the ability to pick and choose the events that take place in the frame sequence. The only restriction on the order of events is that there must be at least one dead time after every rotation. This is because the design emphasizes the use of dead times to keep track of the sequence in testing CCDs.

The option allows any combination of the three $\mathrm{CCD}$ registers to be rotated at a time. In addition, any number of rotation-dead time pairs can occur in succession. This provides easy implementation of actions like frame or register cleaning, register over clocking, pixel addition, and combining CCD registers to form bigger ones. The option accommodates detectors of various array sizes.

The seven different program codes used for the MODIS-T CCDs are shown in Table 3 . Table 4 (a\&b) shows the hex code program used in operating the $4 \times 16 \mathrm{CCD}$ arrays.

\section{Simulation}

Valid's ValidSIM ${ }^{\mathrm{TM}}$ program was used in conjunction with GEDTM to perform digital simulation of the driver. The circuit was verified functionally with the master clock running at $10 \mathrm{MHz}$. Figure 2, a through $\mathrm{c}$, shows the simulation plots of the $C$ Register Driver electrode states for 4,8 , and 16 phase clocking. The plots represent the clocking during a $C$ register rotation. The plots include the PIXEL (one clock cycle delay) and RESET signals in relation to the rotation taking place.

It can be noted how the two outside zeros in each pixel walk through the Register Drivers shift register. Also noted is that, because the electrodes are clocked at the same frequency, it takes 2 and 4 times longer for the 8 and 16 phase CCDs to complete a rotation than in the 4 phase configuration. This means that faster pixel rates are possible with 4 phase clocking.

The fastest pixel rates available with this driver hardware are 400, 350, and $275 \mathrm{~K}$ pixels per second for the 4, 8 , and 16 phase

Table 3: Seven Program Codes Used in Frame Sequence Programs

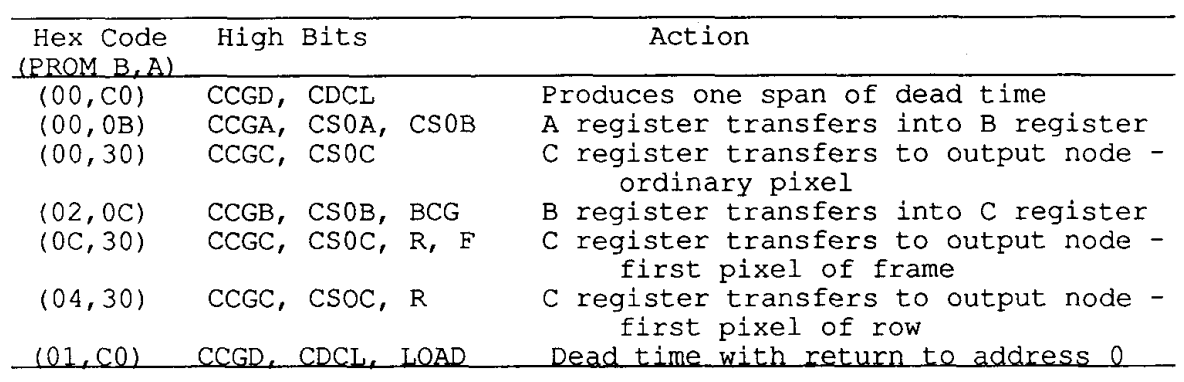


Table 4: PROM Program for 4×16 CCD Arrays a: Contents of PROM A

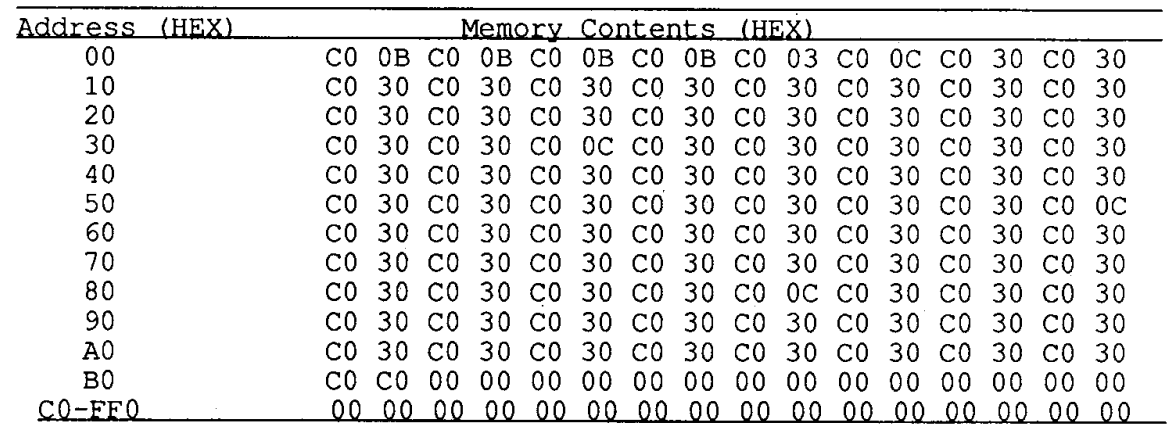

b: Contents of PROM B

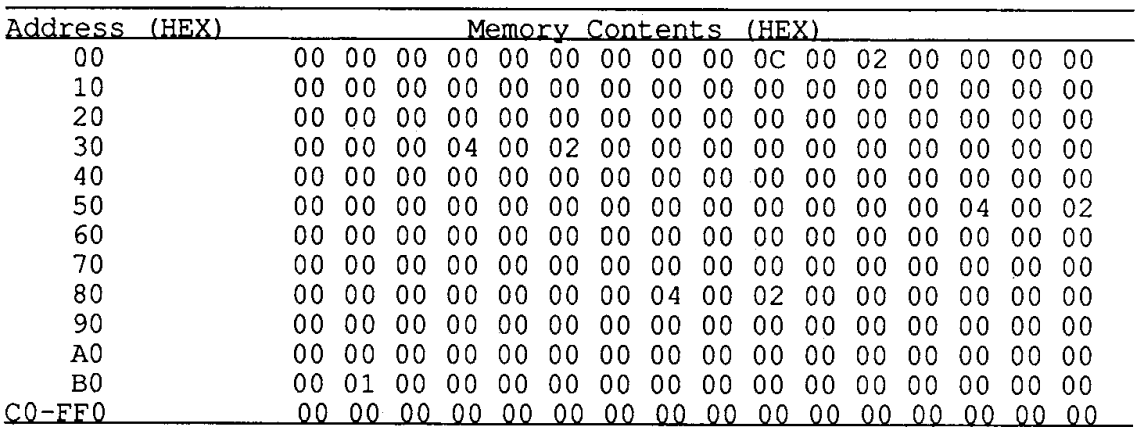

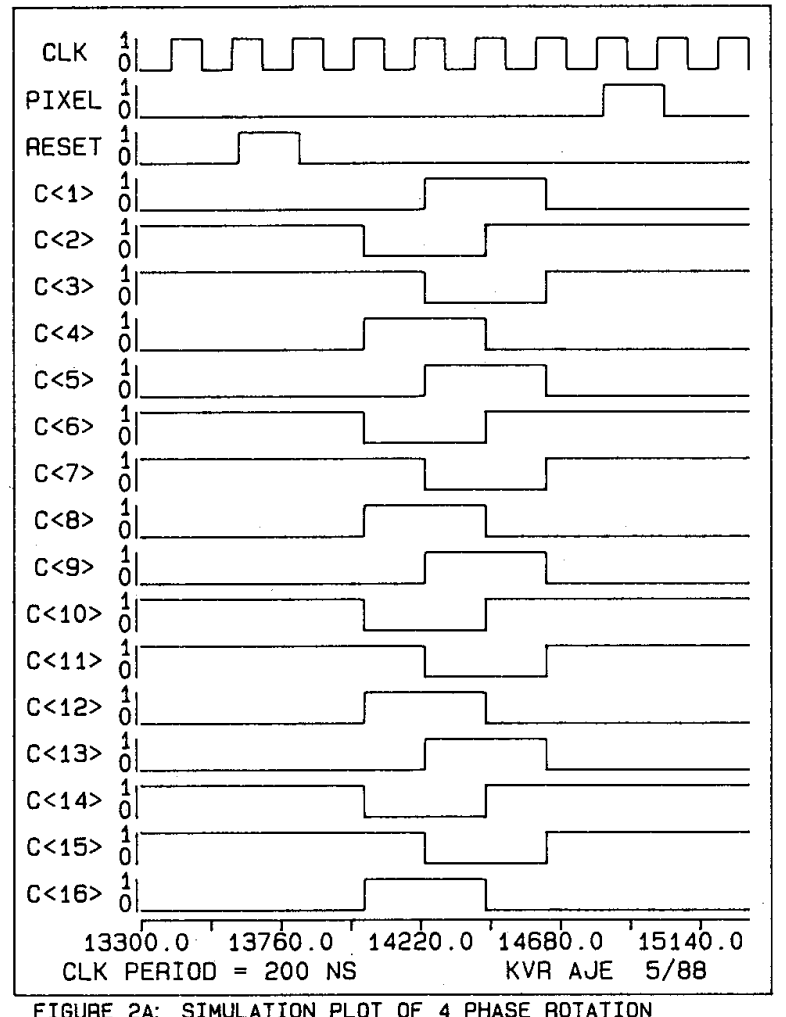

FIGUAE 2A: SIMULATION PLOT OF 4 PHASE ROTATION

cases, respectively. Modifying the hardware by changing the amount of clock cycles per dead time could produce 4 phase clocking rates in the order of $1 \mathrm{M}$ pixels per second.

Figure 3 is the simulation plot of output control signals for an entire frame with 4 rows of 20 pixels. This frame sequence is

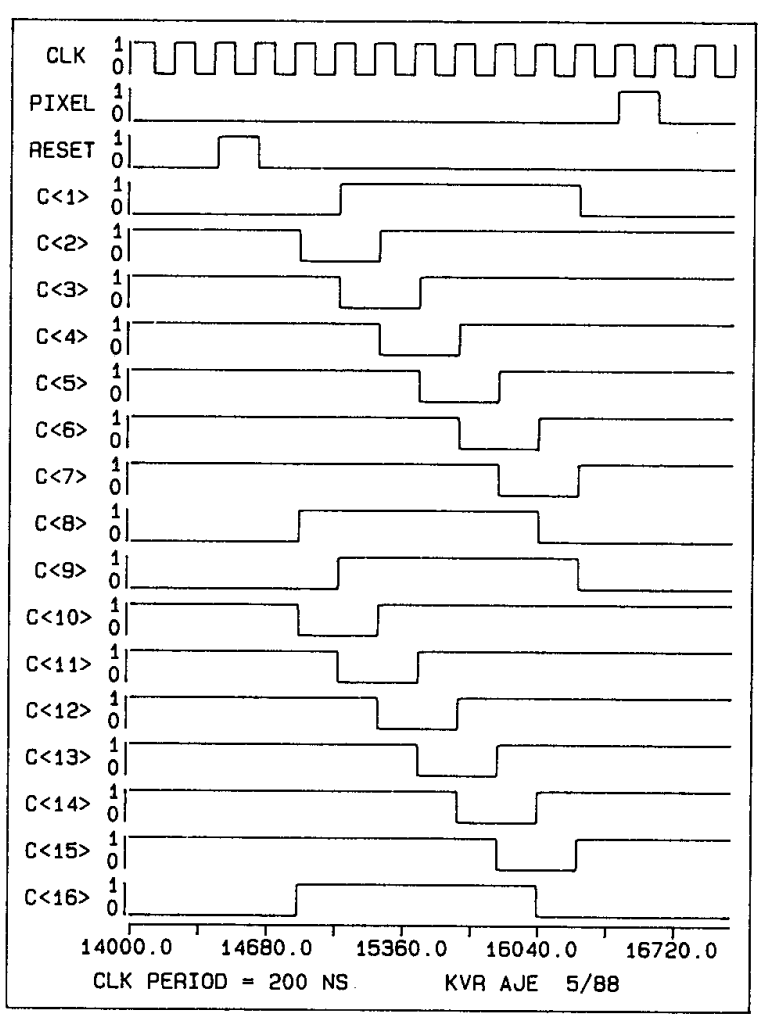

FIGURE 2B: SIMULATION PLOT OF 8 PHASE ROTATION

used to drive the $4 \times 16$ MODIS-T CCDs. The 20 pixels per row provides four over clocked pixels per row worth of data representing the dark pixel voltage level. This voltage is used as a reference level when analyzing the illuminated (image) pixels.

All of the control signals, except BCG, 


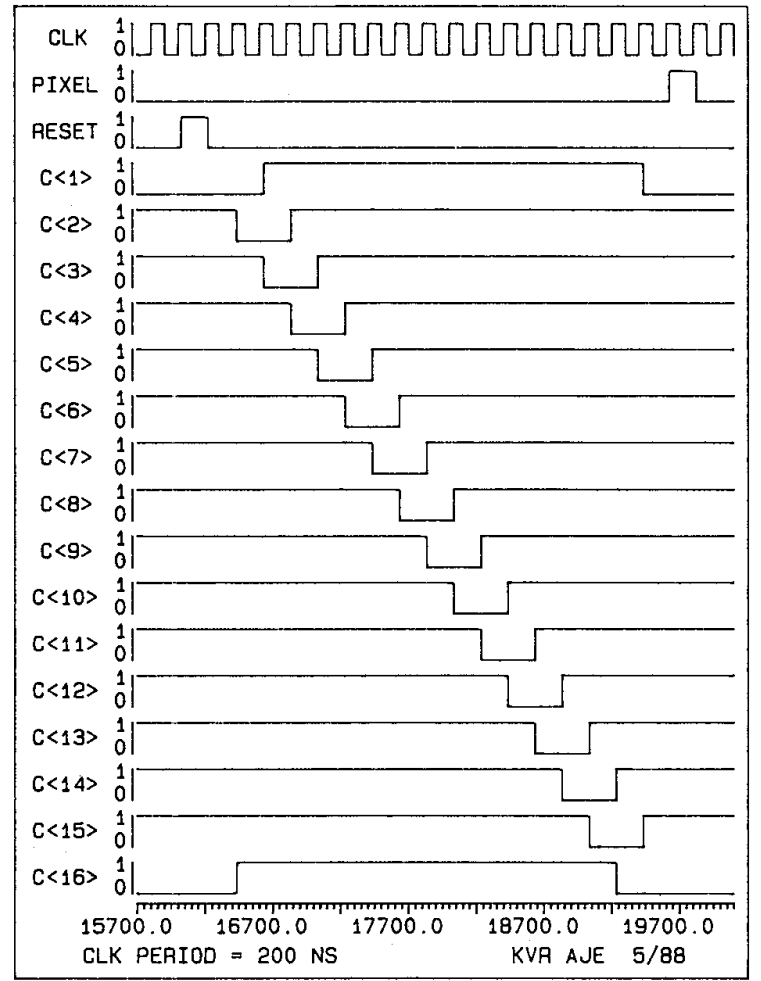

FIGURE 2C: SIMULATION PLOT OF 16 PHASE ROTATION

have pulses of 100 nsec. duration. The BCG signal must be high during an entire rotation to transfer charges between the $B$ register and the $\mathrm{C}$ register. This is seen at the beginning of every row in Figure 3 .

\section{Timing and Packaging Analysis}

The driver design was run through Valid's ValidTIMETM and PACKAGER TM programs before fabrication. The timing analyzer indicated no errors relevant to the driver design. Fan-out problems, found by PACKAGER ${ }^{\mathrm{TM}}$, were fixed and then verified. The driver design uses 54 TTL parts.

\section{Hardware}

The use of valid's design tools, especially the simulator, provided much confidence in creating the working hardware. Two versions of the driver were fabricated: wirewrap and printed circuit board (PCB). The wirewrap version was built as a prototype for the PCB. Both versions were functionally verified with a Tektronix logic analyzer.

The major difference between hardware versions are the capacitors connected between $\mathrm{V}_{\mathrm{CC}}$ and GND near every chip on the PCB. The absence of this on the wirewrap version limits it's maximum operating frequency to a $1 \mathrm{MHz}$ master clock. The PCB, however, operates with a $10 \mathrm{MHz}$ master clock, which agrees with the simulation results.

The PCB also has the option switches mentioned in the Modes of Operation section of this paper. Fabrication of the wirewrap version with switches was not feasible.

The driver uses two DC power supplies. The $V_{C C}$ supply puts out +5 VDC at about $2.5 \mathrm{~A}$. The $+\mathrm{V}$ voltage is normally between $0 \mathrm{~V}$ and +20 VDC and draws about $800 \mathrm{~mA}$.

\section{Practical Modifications}

The MODIS-T CCD Driver design was guided by the special requirement of having the ability to drive 4,8 , or 16 phase CCDs. The unusual requirement stems from the preliminary MODIS-T detector array specifications. The requirement presented a challenge in having to deal with so many clocked signals at once.

Using shift registers yields its self to configurations with any number of phases (especially a large number of phases). The great advantage to this design is the ability to control when a register rotates. There are many things that can be modified to meet various CCD needs. Here are a few:

- Add an'option switch to choose duration of a span of dead time.

- Use buffer drivers, like TSC427s, that do not require pullup resistors.

- Provide a different voltage supply for

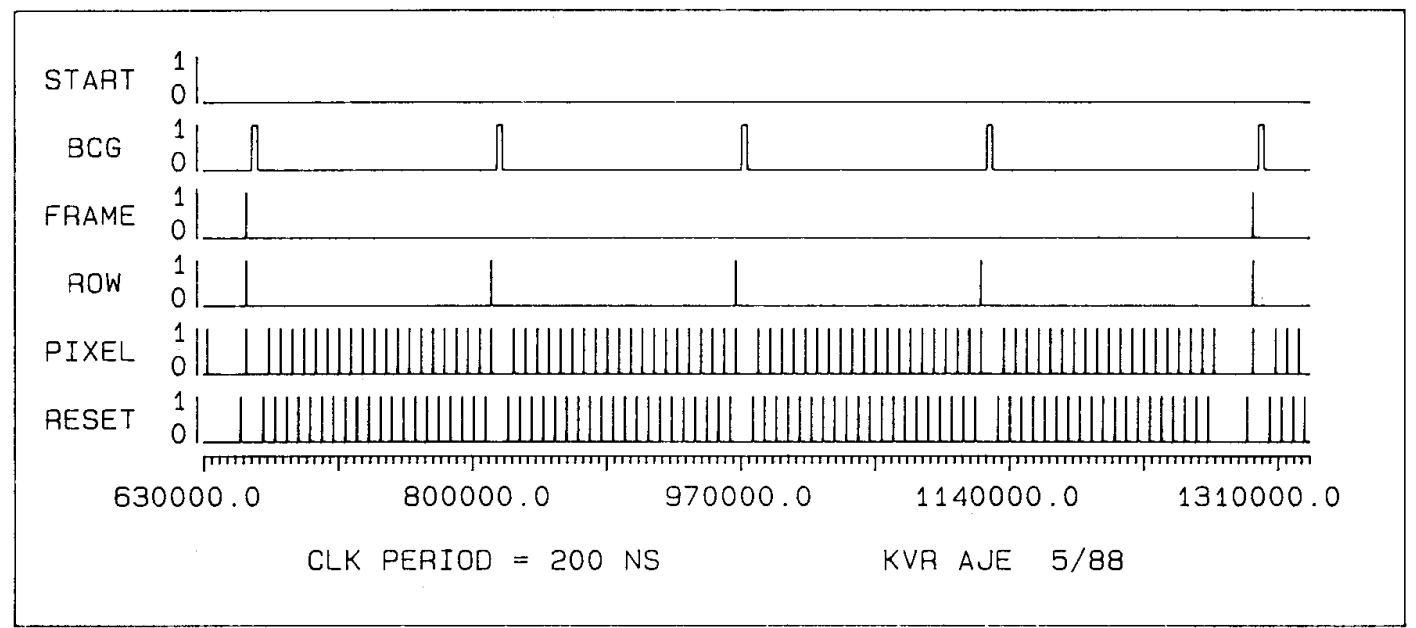

FIGURE 3: SIMULATION PLOT OF 16 PHASE CCD DAIVER

DURING ONE FRAME SEQUENCE

(ONE FRAME WITH 4 ROWS OF 20 PIXELS EACH) 
each set of $\mathrm{CCD}$ register drivers and each CCD control signal.

- Implement a small modification within the control Logic block to allow consecutive rotations in the frame sequence.

- Modify the Addresser logic to select from several frame programs stored in a single PROM set.

- Add Register Driver blocks for solid state devices with more than three CCD registers.

- Add a logic block that provides a simple implementation of special control signals to be independent but working with the other logic blocks.

- Use programmable logic devices to implement certain functions to minimize the number of components.

\section{Acknowledgements}

The authors would like to acknowledge the following people for their contributions in designing, fabricating, and testing the CCD driver.

$\begin{array}{lll}\text { NASA/GSFC: } & \begin{array}{l}\text { Pete Shu } \\ \text { Brent Mott }\end{array} & \text { Murzy Jhabvala } \\ & \text { John McHale Bokerson } \\ \text { STX: } & \text { Fred Minetto Jeff Bowser } \\ \text { JPI: } & \text { Jim Janesick }\end{array}$

\section{References}

[1] C.H. Séquin and M.F. Tompsett, charge Transfer Devices, New York: : Academic Press, 1975, Ch. 2, pp.6-18.

[2] C.H. Séquin and M.F. Tompsett, charge Transfer Devices, New York: : Academic Press, 1975, Ch. 5, pp.152-157. 